\title{
Capsule Commentary on Davydow et al., Depression and Risk of Hospitalizations for Ambulatory Care-Sensitive Conditions in Patients with Diabetes
}

\author{
Matthew J. Bair, MD, MS \\ Roudebush VA Center of Excellence on Implementing Evidence Based Practice, Indiana University School of Medicine, and Regenstrief Institute, \\ Indianapolis, IN, USA.
}

$\mathrm{J}$ Gen Intern Med 28(7):951

DOI: $10.1007 / \mathrm{s} 11606-013-2365-9$

() Society of General Internal Medicine 2013

$\mathrm{H}$ ospitalizations for ambulatory care sensitive conditions (ACSCs) are potentially preventable, assuming timely and appropriate primary care is delivered to outpatients with these common, chronic conditions (diabetes mellitus, hypertension, asthma, COPD). Health care systems and hospitals frequently track and analyze ACSC hospitalizations as a performance measure. ${ }^{1}$ If hospitalization rates are disproportionately high, it is presumed that there may be problems in access to primary care or that outpatient management of these conditions is inadequate.

In this issue of JGIM, Davydow and colleagues ${ }^{2}$ analyzed prospective cohort data in a large sample of patients $(N=4,128)$ with diabetes seen in 9 Group Health Cooperative primary care clinics in the Seattle area. The primary study question was whether comorbid major depression was independently associated with hospitalizations for ambulatory care-sensitive conditions (ACSCs) in patients with diabetes. After adjusting for demographic, clinical, and health-risk behavioral factors, major depression was associated with both the risk of initial ACSC-related hospitalization and the number of hospitalizations due to ACSCs. The hazard ratio and relative risk for ACSC-related hospitalization and the number of hospitalizations was increased by $41 \%$ and $37 \%$, respectively. These important findings suggest that major depression may be an important contributor to hospitalizations for ACSCs.

A limitation of this rigorously conducted study was the lack of information regarding depression treatment among study subjects, e.g. the proportion receiving treatment, the type and effectiveness of treatment and patient adherence and engagement with treatment. Even if these patients were receiving and adherent to depression treatment, it is safe to say that treatment was ineffective, given that participants' scores on the PHQ-9 depression scale ${ }^{3}$ were in the diagnostic range for active, major depression. Despite this limitation, the study has important clinical implications. The findings suggest that improving depression care may be one strategy to prevent or reduce hospitalizations due to ACSCs, and subsequent hospitalization-related costs in patients with comorbid diabetes and depression. As the authors point out, "additional research is warranted to ascertain if effective interventions for depression in patients with diabetes could reduce the risk of hospitalizations for ACSCs and their associated adverse outcomes."

Conflict of Interest: The author declares that he/she does not have a conflict of interest.

Corresponding Author: Matthew J. Bair, MD, MS; Roudebush VA Center of Excellence on Implementing Evidence Based Practice, Indiana University School of Medicine, and Regenstrief Institute, Indianapolis, IN 46202, USA (e-mail: mbair@iupui.edu).

\section{REFERENCES}

1. AHRQ Quality Indicators-Guide to Prevention Quality Indicators: Hospital Admission for Ambulatory Care Sensitive Conditions. Rockville, MD: Agency for Healthcare Research and Quality. Revision 4. (November 24, 2004). AHRQ Pub. No. 02-R0203.

2. Davydow DS, Katon WJ, Lin EHB, Ciechanowski P, Ludman E, Oliver M, Von Korff M. Depression and Risk of Hospitalizations for Ambulatory Care-Sensitive Conditions in Patients with Diabetes. J Gen Intern Med. doi:10.1007/s11606-013-2336-1.

3. Kroenke K, Spitzer RL, Williams JB. The PHQ-9: validity of a brief depression severity measure. J Gen Intern Med. 2001;16:606-613. 